\title{
Twotype multiagent game for egress congestion
}

\author{
Anton von Schantz, Harri Ehtamo \\ Aalto University School of Science \\ \{anton.von.schantz, harri.ehtamo\}@aalto.fi
}

\author{
Ilmari Pärnänen \\ Capgemini Finland Oy \\ ilmari.parnanen@capgemini.com
}

\begin{abstract}
Our starting point is a recently introduced spatial multiagent game for egress congestion. We present a twotype extension of the game. In the game, the agent chooses its strategy by observing its neighbors' strategies. The agent's reward structure depends on its distance to the exit and available safe egress time $\left(T_{A S E T}\right)$. Although $T_{A S E T}$ is a well-defined physical quantity, it is assumed that the agents interpret it subjectively: it is assumed that there are high $T_{A S E T}$ and low $T_{A S E T}$ agent types. Also, we apply the game to a cellular automaton (CA) evacuation model. We show that high $T_{A S E T}$ agents are on average able to overtake low $T_{A S E T}$ agents. However, the more there are high $T_{A S E T}$ agents in the crowd, the more the evacuation becomes inefficient for the whole crowd.
\end{abstract}

\section{Introduction}

In the faster-is-slower effect, the attempt of individuals to evacuate faster results in a slower evacuation for the whole crowd. In [5] the phenomenon was studied experimentally. A group of people were set to evacuate in two different scenarios, one where people tried to evacuate faster by pushing their way to the exit, and another where pushing was forbidden. It was shown that when the individuals tried to evacuate faster, the evacuation for the whole group become slower. Contradicting experimental results have been presented in [8]. However, the results of these two experiments are not directly comparable with each other due to differences in incentive systems as well as in the exit types of the test geometry.

In [6] Helbing gave a physical explanation to the fasteris-slower effect. When a crowd tries to evacuate through a bottleneck, the back of the crowd push the agents in front of them. The driving force of the back of the crowd, combined with the frictional force between the agents, causes human arches to build up. The arches break down when there are large enough fluctuations in the forces. These arches not only slow down the evacuation, but people are squeezed and suffocated in them.

An indicator of the severity of the arch-formation is the distribution of time lapses between consecutive evacuating people. The stronger the human arches are, the more probable are longer time lapses between consecutive evacuating people. In [5] it was shown that in the scenario, where people tried to evacuate faster, the probability for longer time lapses was higher. It was also speculated, that if the situation is competitive enough, the expected value for the time lapse distribution might not converge. This means that rare disastrous events are possible.

A common explanation for the self-destructive behavior of a crowd is that people are panicking. However, sociologists have been unanimous for decades that panic doesn't occur in crowds, and that panic is merely a term for a poorly understood phenomenon [10]. Because of ethical concerns, there is little experimental research on the decision-making of people in fire emergencies. Nonetheless, there are experiments, where a fire emergency has been simulated by using an analogous reward structure to that in competitive fire egress $[11,13,14]$. In the experiments, it was shown that the faster-is-slower effect is a result of rational behavior. If individuals in a fire emergency are considered rational, their decision-making can be modeled with game theory $[2,3,7,9]$.

Heliövaara et al. [7] use the spatial game approach to model the decision-making of agents in an egress congestion. The agents have two different behaviors, or strategies to choose, Patient and Impatient. They observe the strategies of the agents in their immediate neighborhood and choose their strategy according to the myopic best response rule. In the game, an agent's reward structure depends on its distance to the exit and available safe egress time $\left(T_{A S E T}\right) . T_{A S E T}$ is a widely used measure in the fire evacuation literature [4]. It describes the time it takes for the fire conditions to become dangerous.

The game model has been coupled to a cellular automaton (CA) evacuation model in $[16,17]$. The CA model is based on the model by Kirchner et al. [12]. In the CA, the agents' positions are updated with simple stochastic update rules. Many emergent phenomena in evacuations, e.g., faster-is-slower effect, clogging at bottlenecks, and herding, can be realistically simulated with a CA $[12,16]$. Also, since the model is discrete in time and space it is computationally light.

The model in [16] is the starting point of our article. In $[7,16]$, it was assumed that all agents have the same value for $T_{A S E T}$. The contribution of this article is to extend the 
game to allow two types of agents in the crowd. Although $T_{A S E T}$ is a well-defined physical quantity, it is assumed that the agents interpret it subjectively: it is assumed that there are high $T_{A S E T}$ and low $T_{A S E T}$ agent types. In Sec. 2 the new game is presented, and in Sec. 3 we examine its equilibria.

Also, we modify the CA model in Sec. 4. We exclude the herding effect, since it is not relevant when we are simulating the evacuation through a single exit. In [16], the probability that none of the agents are allowed to move in a situation, where several agents try to move to the same cell, was modeled with the friction parameter $\mu$. It was held constant throughout the simulation. Here, we set $\mu$ to depend on the density of the crowd and proportion of impatient agents in the crowd.

We are interested in how the agent's subjective interpretation of the fire threat, i.e., the agent's type affects the efficiency of the evacuation on both individual and crowd level. Thus, in Sec. 5, we study the evacuation efficiency of high $T_{A S E T}$ and low $T_{A S E T}$ agents. We are interested in their efficiency on both individual and crowd level. We examine both evacuation time, and the distribution of time lapses between consecutive agents.

\section{Game-theoretical model}

Next, we present a twotype spatial multiagent game for egress congestion. It should be mentioned, that the game only describes what the agents expect that will happen in different encounters. The agents' decision-making is based on these expectations. We do not assume that the agents can take into account the full complexity of all interacting agents. Hence, when coupling the game with the CA model in Sec. 4, the actual outcome of an interaction is not necessarily exactly what the agents expected when selecting their strategies.

The game models a competitive egress from a room with a single exit. In the game, there are $n$ agents, indexed by $i, i \in I=\{1, \ldots, n\}$. The agents are located in a room, which is discretized into a square grid, so that a single agent occupies a single cell of the grid.

Each agent has an estimated evacuation time $T_{i}$. Agent $i$ estimates it by calculating

$$
T_{i}=\frac{\lambda_{i}}{\beta}
$$

Here, $\lambda_{i}$ is the number of agents closer to the exit than agent $i$, and $\beta$ is the capacity of the exit. Since the agents are packed in front of the exit, the walking time to the exit is assumed to be much smaller in comparison to the queuing time. Thus, the agents assume $T_{i}$ equals only the time it takes to queue to the exit. In a contest between two nearby agents $i$ and $j$, their estimated evacuation times are approximated $T_{i j}:=\left(T_{i}+T_{j}\right) / 2$.

Each agent has a cost function $u\left(T_{i j}\right)$, for which $u^{\prime}\left(T_{i j}\right) \geq 0$. The derivative condition describes the fact that it is more costly, i.e., there is a larger risk to be exposed to fire related harm, if the estimated evacuation time $T_{i j}$ is large. Assuming that $\triangle T$ is small we can approximate,

$$
\triangle u\left(T_{i j}\right)=u\left(T_{i j}-\triangle T\right)-u\left(T_{i j}\right) \cong u^{\prime}\left(T_{i j}\right) \triangle T .
$$

The agents have two strategies to choose from: Patient and Impatient. It is assumed that in an actual play of the game, these strategies correspond to patient and impatient behaviors of the agents, respectively. Denote by $N_{i}$ the neighborhood of agent $i$; it will be specified later. In a contest between two neighboring agents $i$ and $j \in N_{i}$, the agents assume the following outcomes:

1. In an impatient vs. patient agent contest, the impatient agent can overtake the patient agent. The impatient agent reduces its estimated evacuation time by $\triangle T$. Thus, the reduction in its cost is $\triangle u\left(T_{i j}\right)$. Because the patient agent's evacuation time is increased by $\triangle T$, its cost is increased by $\triangle u\left(T_{i j}\right)$.

2. In a patient vs. patient agent contest, the patient agents do not compete with each other, they keep their positions and their costs do not change.

3. In an impatient vs. impatient agent contest, neither agent can overtake the other, but they will face a conflict and have an equal chance of getting injured. The risk of injury is described by a cost $C>0$, which affects both agents. The constant $C$ is called the cost of conflict.

From the aforementioned assumptions, a $2 \times 2$ game matrix is constructed

\begin{tabular}{c|c|c|}
\multicolumn{1}{c}{ Impatient } & Patient \\
\cline { 2 - 3 } Impatient & $C, C$ & $-\triangle u\left(T_{i j}\right), \triangle u\left(T_{i j}\right)$ \\
\cline { 2 - 3 } Patient & $\triangle u\left(T_{i j}\right),-\triangle u\left(T_{i j}\right)$ & 0,0 \\
\cline { 2 - 3 } &
\end{tabular}

In the game matrix, agent $i$ is a row player and agent $j$ a column player. When a particular pair of strategies is chosen, the costs for the two agents are given in the corresponding cell of the matrix. The cost to agent $i$ is the first number in a cell, followed by the cost to agent $j$. Because this is a cost matrix, the agents want to minimize their outcome in the game. 


\subsection{Cost function}

In the fire safety literature, available safe egress time $T_{A S E T}$ is a widely-used physical measure for the time it takes for the fire conditions to become lethal [4].

Often computer simulations are needed to assess $T_{A S E T}$ accurately [4]. Thus, it is hardly conceivable that humans, that are under time pressure and have limited cognitive abilities [15] could accurately estimate $T_{A S E T}$. In other words, it is very unlikely that humans have a realistic perception of the threat of the situation. Probably, some of them consider the situation more threatening than others. In our game model, we assume the agents to have a subjective measure of $T_{A S E T}$. We assume that there are two types of agents in the crowd, high $T_{A S E T}$ agents and low $T_{A S E T}$ agents. We denote their types by $T_{A S E T}^{H}$ and $T_{A S E T}^{L}$, respectively. Thus, for the type variable it holds $T_{A S E T} \in\left\{T_{A S E T}^{H}, T_{A S E T}^{L}\right\}$.

Let us now go back to Eq. (2). If we for simplicity assume $\triangle T=1$, we have $\triangle u\left(T_{i j}\right) \cong u^{\prime}\left(T_{i j}\right)$. So, the cost of being overtaken is approximately $u^{\prime}\left(T_{i j}\right)$. Let's make another assumption about $u\left(T_{i j}\right)$. Recall, that $u^{\prime}\left(T_{i j}\right) \geq 0$. We additionally assume that $u^{\prime \prime}\left(T_{i j}\right) \geq 0$, i.e., an agent considers the cost of being overtaken larger if the estimated evacuation time $T_{i j}$ is larger. Also, we assume that when $T_{i j}=T_{A S E T}$, the agent is indifferent between being overtaken or risking an injury in a conflict against another impatient agent, i.e., $u^{\prime}\left(T_{A S E T}\right)=C$.

Now we can give an explicit form to the cost function $u\left(T_{i j}\right)$. An example of a cost function that fulfills these conditions is

$$
u\left(T_{i j}\right)=\left\{\begin{array}{cl}
\frac{C T_{i j}^{2}}{2 T_{A S E T}}, & \text { if } T_{i j} \geq 0, \\
0, & \text { if } T_{i j}<0 .
\end{array}\right.
$$

In Fig. 1, the cost functions for high $T_{A S E T}$ agents $\left(T_{A S E T}^{H}=1000\right)$, and low $T_{A S E T}$ agents $\left(T_{A S E T}^{L}=400\right)$ are depicted.

For both cost functions, the cost of conflict parameter is set $C=3$. From the figure, it can be seen that for the low $T_{A S E T}$ agent, the cost function grows faster. Now, substitute $\triangle u\left(T_{i j}\right)=u^{\prime}\left(T_{i j}\right)$ in the game matrix, and divide it by $u^{\prime}\left(T_{i j}\right)$. This does not affect the equilibria of the game. Finally, substitute $u^{\prime}\left(T_{i j}\right)=T_{i j} / T_{A S E T}$. Then, we get the following game matrix

Notice how the game now only depend on the parameter $T_{A S E T} / T_{i j}$. Whether agents $i$ and $j$ are of same or different type, the game is either symmetric or asymmetric, respectively. In a symmetric game, the costs only depends on the strategies chosen by the agents, not on which agent is playing them. In an asymmetric game, the costs also depend on which type of the agents is playing the strategies. For general comments on 2 player $2 \times 2$ games see

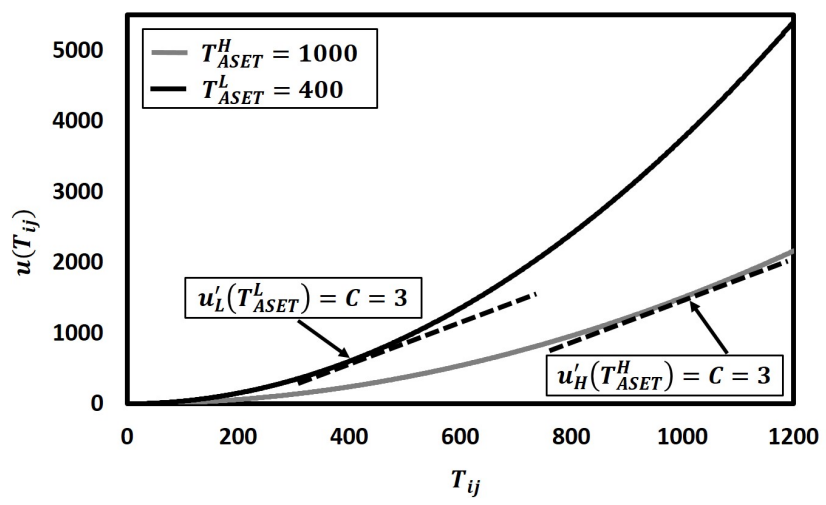

Figure 1: Cost functions for high $T_{A S E T}$ agents $\left(T_{A S E T}^{H}=1000\right)$, and low $T_{A S E T}$ agents $\left(T_{A S E T}^{L}=400\right)$.

Impatient Patient

\begin{tabular}{c|c|c|}
\cline { 2 - 3 } Impatient & $\frac{T_{A S E T}}{T_{i j}}, \frac{T_{A S E T}}{T_{i j}}$ & $-1,1$ \\
\cline { 2 - 3 } Patient & $1,-1$ & 0,0 \\
\hline
\end{tabular}

the appendix in [7].

In the symmetric case, when $0<T_{A S E T} / T_{i j} \leq 1$, the game played is Prisoner's Dilemma, PD, and the only Nash equilibrium (NE) is (Impatient, Impatient). The only NE of PD is an evolutionary stable strategy (ESS) [18]. And when $T_{A S E T} / T_{i j}>1$, the game played is Hawk-Dove, $\mathrm{HD}$, and there are two pure strategy Nash equilibria (Impatient, Patient) and (Patient, Impatient). There is also a mixed strategy equilibrium, where the strategy Impatient is played with probability $T_{i j} / T_{A S E T}$, and the strategy Patient with probability $1-T_{i j} / T_{A S E T}$. The mixed strategy equilibrium is an ESS.

In the asymmetric case, it holds for agent $i, T_{A S E T}=$ $T_{A S E T}^{H}$, and for agent $j, T_{A S E T}=T_{A S E T}^{L}$, or vice versa: if $0<T_{A S E T}^{H} / T_{i j}, T_{A S E T}^{L} / T_{i j} \leq 1$, the game played is an asymmetric PD, and the only NE is (Impatient, Impatient), which is an ESS; and, when $T_{A S E T}^{H} / T_{i j}>1$ and $0<T_{A S E T}^{L} / T_{i j} \leq 1$, the game played is less-known in the game theory literature, but it is sometimes called the game of Samson and Delilah. From hereon, we will call this instance of the game Samson. In Samson, the only NE, which is an ESS, is where the high $T_{A S E T}$ agent plays Patient and the low $T_{A S E T}$ agent plays Impatient. Lastly, if $T_{A S E T}^{H} / T_{i j}, T_{A S E T}^{L} / T_{i j}>1$, the game played is an asymmetric HD, and there are two pure strategy NE (Impatient, Patient) and (Patient, Impatient). There is also a mixed strategy equilibrium, where the high $T_{A S E T}$ agent plays Impatient with probability $T_{i j} / T_{A S E T}^{L}$, and Patient with proba- 
bility $1-T_{i j} / T_{A S E T}^{L}$, and the low $T_{A S E T}$ agent plays Impatient with probability $T_{i j} / T_{A S E T}^{H}$, and Patient with probability $1-T_{i j} / T_{A S E T}^{H}$. The pure strategy NE's are ESS's.

\subsection{Update of strategies}

Next, we present how the agents update their strategies. The agents play the game with the agents in their Moore neighborhood, i.e., the agents in the 8 nearest cells. We denote the set of agents in the Moore neighborhood of agent $i$ by $N_{i}$. Notice that when we later set the agents to move, the neighboring agents will change as the agents move.

The game is played over periods $t=0,1, \ldots$. Agent $i$ 's strategy on period $t$ is denoted by $s_{i}^{t}$. Here, $s_{i}^{t} \in S$ and $S=$ \{Patient, Impatient $\}$. The agents are assumed to update their strategies according to the shuffle update scheme, i.e., during $n$ periods each of the agents updates its strategy once. The order in which the agents update their strategies is randomly chosen.

The agents' objective is to minimize the sum of costs against their neighbors. The agents are assumed to update their strategies with the myopic best-response rule, i.e., agent $i$ observes the current strategies of its neighbors in $N_{i}$, not considering past or future play, and chooses its best-response strategy. The best-response strategy of agent $i$ is denoted by $B R_{i}$. Note that the types of the neighboring agents doesn't affect $B R_{i}$. The reason for this is that the agent's type is its private information, which we assume the other agents can't observe.

Let us assume the initial strategy profile of the agents to be $s^{0}=\left(s_{1}^{0}, \ldots, s_{n}^{0}\right)$. On period $t$ agent $i$ chooses its strategy as follows

$$
\begin{aligned}
s_{i}^{(t)} & =B R_{i}\left(s_{-i}^{(t-1)} ; T_{i}, T_{-i}\right) \\
& =\underset{s_{i}^{\prime} \in S}{\arg \min _{j \in N_{i}}} v_{i}\left(s_{i}^{\prime}, s_{j}^{(t-1)} ; T_{i j}\right) .
\end{aligned}
$$

Here, the notation $s_{-i}^{(t-1)}$ is used to denote the strategies of all other agents than agent $i$ at period $t-1$, and $T_{-i}$ includes the estimated evacuation times of these agents. The function $v_{i}\left(s_{i}^{\prime}, s_{j}^{(t-1)} ; T_{i j}\right)$ gives the cost defined by the previously introduced game matrix to agent $i$, when it plays strategy $s_{i}^{\prime}$, and agent $j$ has played strategy $s_{j}^{(t-1)}$ on period $(t-1)$

The simplicity of the myopic best-response rule reflects the limited cognitive abilities that humans are assumed to have in a stressful situation [15]. Also, it is unrealistic to assume that the agents updated their strategies simultaneously. In many real social systems the agents update their strategies independent of each other [19]. Thus, the shuffle update scheme is more suitable for strategy update.

\section{Equilibrium analysis}

In a Nash equilibrium of the spatial game, none of the agents can lower its cost by unilaterally deviating from its equilibrium strategy. In $[7,16]$ the equilibria of the spatial multiagent game for egress congestion with a single agent type was analyzed. There, given any initial configuration of strategies, the game always converges to an equilibrium, when all the agents have updated their strategies about 10 times with the shuffle update scheme. For a certain sized crowd, with a specific value for $T_{A S E T}$, there are multiple equilibria, i.e., the strategies can be distributed in many ways in the square grid, so that no agent can lower its cost by unilaterally deviating. However, the proportion of impatient agents is the same in each of these equilibria.

Next, we analyze the equilibria of the twotype spatial multiagent game for egress congestion. where 1498 agents have been set to play the game until equilibrium is reached. In an egress congestion, people typically organize into a half-circle-like formation in front of the exit. Thus, the agents are set into a half-circle in front of the exit. We set $T_{A S E T}^{H}=1000$ and $T_{A S E T}^{L}=400$. In Fig. 2a all agents are high $T_{A S E T}$, in Fig. $2 \mathrm{~b}$ all agents are low $T_{A S E T}$, and in Fig. $2 \mathrm{c}$, we consider a crowd with $50 \%$ high $T_{A S E T}$ agents and $50 \%$ low $T_{A S E T}$ agents. In Fig. 2, the equilibrium configurations of these three scenarios are shown. Black squares represent impatient agents and gray squares represent patient agents. The half-circles divide the area into subareas, where different games are played.

As expected, the strategies of the agents in Figs. $2 \mathrm{a}$ and $2 \mathrm{~b}$ converged to an equilibrium. In Fig. $2 \mathrm{a}$, we can distinguish between two areas. They are separated by a black curve. In the area inside the black curve, all the agents play HD. Outside the black curve, the estimated evacuation time $T_{i j}$ is so large in comparison to $T_{A S E T}$ that the agents play PD. Thus, all agents outside the black curve are impatient. This area is denoted with $C$. In Fig. 2b, $T_{A S E T}$ is lower in comparison to Fig. 2a. Thus, the agents are more threatened by the situation, and a higher proportion of agents are impatient, in comparison to Fig. 2a. This results in the agents playing PD much closer to the exit. Thus, the black curve is much lower here than in Fig. 2a. The area in Fig. 2b, where the agents play HD is denoted by $A$.

Interestingly, also in Fig. 2c, where there are two type of agents, the strategies converge to equilibrium, when the agents have updated their strategies about 10 times with the shuffle update scheme. In the equilibrium, we can distinguish between 3 different areas. In area $A$, agents of the same type play $H D$ against each other, and agents of different type asymmetric $H D$ against each other. In area $C$, agents of the same type play $P D$ against each other, and agents of different type asymmetric $P D$ against each other. 


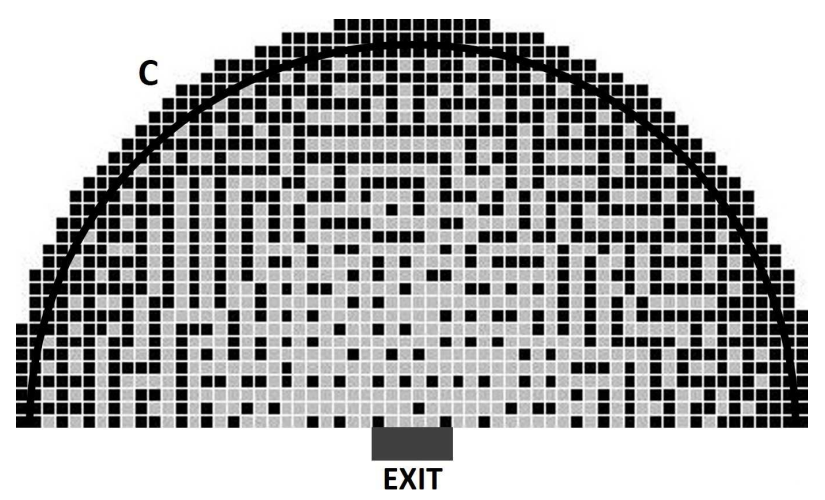

(a)

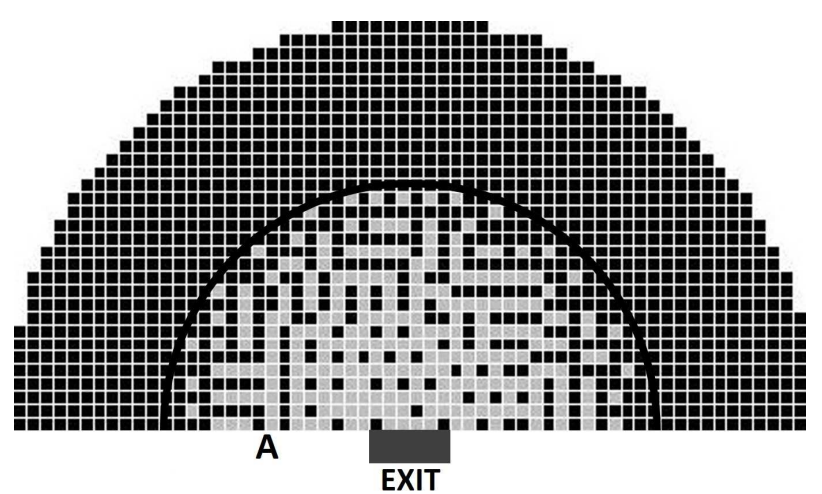

(b)

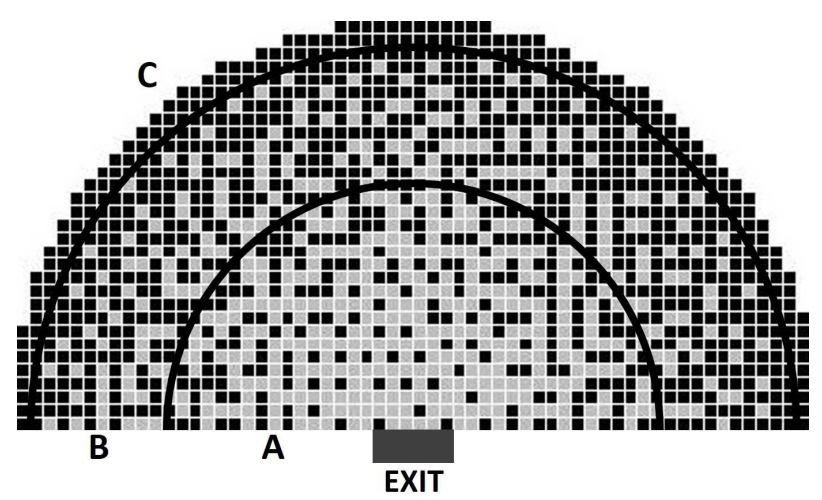

(c)

Figure 2: Equilibrium configurations, where (a) all agents are high $T_{A S E T}$, (b) all agents are low $T_{A S E T}$, (c) $50 \%$ are high $T_{A S E T}$ agents and other $50 \%$ low $T_{A S E T}$ agents. Black squares represent impatient agents and gray squares represent patient agents. The half-circles divide the area into subareas, where different games are played.

In between these two areas is area $B$, where high $T_{A S E T}$ agents play HD against each other, low $T_{A S E T}$ agents play $P D$ against each other, and agents of different type play the game Samson against each other.
A surprising effect can be noticed, by comparing the proportion of impatient agents in the different scenarios of Fig. 2. In the single type crowd, in Fig. 2a, ca. $60 \%$ of high $T_{A S E T}$ agents are impatient, whereas, in the twotype crowd, in Fig. 2 c, only $40 \%$ of high $T_{A S E T}$ agents are impatient. However, the opposite effect does not happen to low $T_{A S E T}$ agents, in both the single and twotype crowds ca. $90 \%$ of the low $T_{A S E T}$ agents are impatient.

This can be explained by examining the agents' bestresponse strategy from Eq. (4), which can be expressed as an inequality. Recall the costs associated with interactions of different strategists. Now, agent $i$ should play Impatient, if the cost of playing Impatient against its neighbors is less than or equal to the cost of playing Patient:

$$
\sum_{j \in N_{i}} \frac{T_{A S E T}}{T_{i j}}+\left(\left|N_{i}\right|-\left|N_{i}^{I m p}\right|\right) \leq\left|N_{i}^{I m p}\right|,
$$

where $\left|N_{i}\right|$ is the number of agents in the neighborhood of $i$ and $\left|N_{i}^{I m p}\right|$ is the number of impatient agents in the neighborhood of $i$. To make the analysis simpler, let us approximate $T_{i}=T_{j}, j \in N_{i}^{\operatorname{Imp}}$. Then the above inequality can be written as

$$
\frac{\left|N_{i}^{I m p}\right|}{\left|N_{i}\right|} \leq \frac{T_{i}}{T_{A S E T}} .
$$

Here, $\left|N_{i}^{I m p}\right| /\left|N_{i}\right|$ is the proportion of impatient agents in the Moore neighborhood of agent $i$. In a spatial equilibrium of the game, Eq. (6) has to hold for all agents $i \in N$. We can infer that in an equilibrium a low $T_{A S E T}$ agent plays Impatient with a higher proportion of impatient agents in its neighborhood compared to a high $T_{A S E T}$ agent. Thus, in a twotype crowd, in an equilibrium, low $T_{A S E T}$ agents are going to fill most of the space for impatient agents.

\subsection{Sensitivity analysis}

Next, the same simulation setup is used as in Fig. 2. We fix the value for $T_{A S E T}^{H}$ and alter the value for $T_{A S E T}^{L}$. Then, we study the proportions of impatient agents in the spatial equilibrium of the game. The simulations for each fixed $T_{A S E T}^{H}$ are run for 100 different values of $T_{A S E T}^{L}$, equally distributed in the interval $\left[0, T_{A S E T}^{H}\right]$. The interpolated curves of the data points are shown in Fig. 3.

For example, see Fig. 3, the curve in the top, there $T_{A S E T}^{H}=500$. The simulations show that when the value for $T_{A S E T}^{L}$ is increased, the proportion of impatient agents in the spatial equilibrium decreases.

In Fig. 4 we examine how altering the proportion of low $T_{A S E T}$ agents in a crowd changes the proportion of impatient agents in the equilibrium. The simulations are run for 100 different proportions of impatient agents, equally 


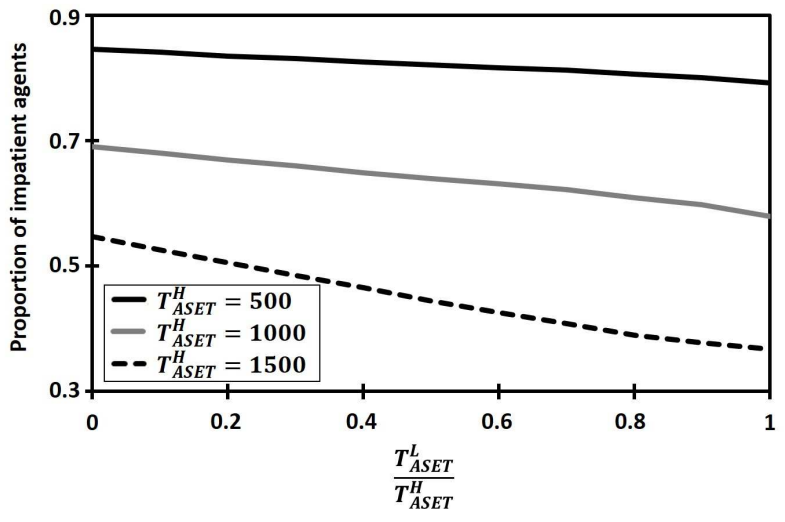

Figure 3: Proportion of impatient agents in the spatial equilibrium, when $T_{A S E T}^{H}$ is fixed and $T_{A S E T}^{L}$ is altered.

distributed between $[0,1]$. Otherwise, same settings are used as in Fig. 2, i.e., $T_{A S E T}^{H}=1000$ and $T_{A S E T}^{L}=400$.

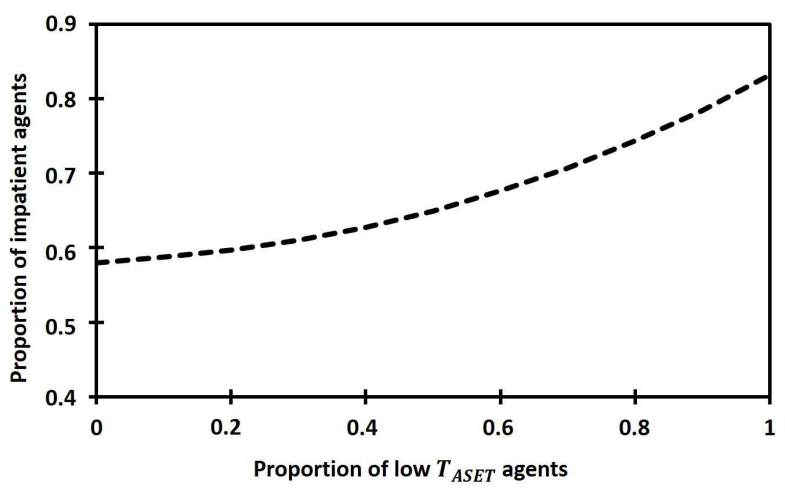

Figure 4: Proportion of low $T_{A S E T}$ agents in the crowd vs. the proportion of impatient agents in the equilibrium of the crowd.

As the proportion of low $T_{A S E T}$ agents in the crowd increases, the proportion of impatient agents in the equilibrium of the game increases.

It should be noted that the size of the crowd also has an effect on the proportion of impatient agents in the equilibrium. Since, when the agents update to their best-response strategy, they consider the ratio $T_{A S E T} / T_{i j}$. Recall Eq. (1); $T_{i j}$ is calculated for an agent by considering the amount of agents closer to the exit than the agent in question. Thus, if the crowd size is increased, the agents in the back of the crowd will have higher values for $T_{i j}$.

\section{Simulation model}

The interactive decision-making situation of individuals in an egress congestion can be modeled with a spatial game as above. Next, we present the CA model, to which the game is coupled. The CA is a modified version of the model used in [16]. In the CA, the agents move in the cells of a discrete square grid. At the beginning of a time step, the agents are allowed to move one cell in orthogonal directions. The dimensions of a cell are assumed to be $0.4 m \times 0.4 m$, and the length of a time step $0.3 \mathrm{~s}$. Movement in diagonal directions is not allowed, since it accounts to a higher velocity. The agents' movement directions are determined by transition probabilities. These transition probabilities are proportional to the static floor field $S F$. The closer the cells are to the exit, the higher values $S F$ attains. In [16] a more complex CA was used, where the agents movement also depended on the so-called dynamic floor field $D F$. The dynamic floor field is omitted here, since its main purpose is to model the herding effect of agents, which is not relevant phenomenon in the evacuation geometries we use in this article.

So, in our model the probability $p_{l m}$ for an agent to move to a neighbor cell $(l, m)$ is calculated as follows

$$
p_{l m}=\frac{1}{Z} e^{k_{S F} S F_{l m}}\left(1-\xi_{l m}\right),
$$

where

$\xi_{l m}= \begin{cases}1 & \text { for forbidden cells (walls and occupied cells) } \\ 0 & \text { else }\end{cases}$ and the normalization

$$
Z=\sum_{(l, m)} e^{k_{S F} S F_{l m}}\left(1-\xi_{l m}\right) .
$$

Here, $k_{S F} \in[0, \infty)$ is the agent's coupling parameter to $S F$. Basically, the larger $k_{S F}$ is, the more straight the agent is moving towards the exit, or the more assertive they are. For a thorough analysis on how the floor fields and coupling parameters affect the movement of the crowd, see $[1,12,16]$.

The agents movement is updated with the parallel update scheme, i.e., all the agents desired movement directions are updated simultaneously. Now, there are situations where several agents desire to move to the same cell, i.e., conflict situations. In [12] a friction parameter $\mu$ was introduced, which describes the probability that none of of the agents in a conflict situation are able to move to the cell. The friction parameter works as a kind of local pressure between the agents [12]. In [12] it was shown that these conflict situations are not just an artifact of the parallel update scheme, but an important feature to describe evacuation dynamics correctly.

\subsection{Friction parameter}

In $[12,16], \mu$ is assumed to be fixed throughout the simulation. However, if $\mu$ is to work as a kind of local pressure between agents, it should vary based on the size of 
the crowd, and on the proportion of impatient agents in it. We define $\mu$ as

$$
\mu=b_{1} \rho_{a} \rho_{\text {Imp }}+b_{2} \rho_{a}+b_{3} \rho_{\text {Imp }} .
$$

Here, $b_{i}, i=1,2,3$ are coefficients for which $b_{1}+b_{2}+$ $b_{3}=1$, and $\rho_{\text {Imp }}$ is the proportion of impatient agents in the crowd. $\rho_{a}$ is calculated by dividing the current size of the crowd with the initial size of the crowd. Thus, in the beginning of the evacuation simulation $\rho_{a}=1$, and as agents are able to evacuate the room, $\rho_{a}$ decreases. This is a reasonable approximation for the effect of crowd size, if the initial crowd is large and the agents evacuate from a room with a single exit. However, for more complex geometries $\rho_{a}$ should be calculated differently.

Since $\rho_{a}, \rho_{\text {Imp }} \in[0,1]$, it holds that $\mu \in[0,1]$. The interaction term $\rho_{a} \rho_{\text {Imp }}$ captures the interaction between crowd size and proportion of impatient agents. We assume the interaction term has the largest impact on $\mu$. For simplicity, we assume that the impact of $\rho_{a}$ and $\rho_{\text {Imp }}$ equally large. This leads us to use the parameter values $b_{1}=0.6, b_{2}=0.2, b_{3}=0.2$ in simulations in our article. We are mainly interested in qualitative phenomena in evacuations. For quantitative accuracy, the correct coefficient values should obviously be estimated from experimental data.

Let us examine how altering the value of a single coefficient $b_{i}, i=1,2,3$, affects the value of $\mu$. If the value for $b_{1}$ is increased, $\mu$ decreases. On the other hand, if $b_{1}$ is decreased, $\mu$ increases. The effect of parameters $b_{2}$ and $b_{3}$ is not so straightforward. If $b_{2}$ is increased, $\mu$ decreases when $\rho_{a}>\rho_{\text {Imp }}$, and $\mu$ increases when $\rho_{a}<\rho_{\text {Imp }}$. For the parameter $b_{3}$ the opposite occurs, if $b_{3}$ is increased, $\mu$ increases when $\rho_{a}>\rho_{\text {Imp }}$, and $\mu$ decreases when $\rho_{a}<\rho_{\text {Imp }}$.

As $\rho_{a}$ and $\rho_{\text {Imp }}$ change during the simulation of an evacuation, altering the coefficients $b_{2}$ and $b_{3}$ can increase $\mu$ at some stages in the simulation and decrease it in others, compared to a simulation done with the values for $b_{i}, i=1,2,3$ used in this article. Thus, altering parameters $b_{2}$ and $b_{3}$ results in nonlinear effects on the evolution of $\mu$ during the simulation.

\subsection{Spatial game coupled with a CA evacu- ation model}

The spatial game is coupled to the CA evacuation model. For technical purposes, the movement of the agents is updated in parallel. Without parallel update, there would be no well-defined time scale. On the other hand, the strategies of the agents are updated with the shuffle update scheme, because it is more realistic to assume that agents do not simultaneously update their strategies.

The time scale in updating strategies is assumed to be much smaller than that of movement. Thus, the crowd is in a spatial equilibrium at a snapshot of the simulation. Yet again, it should be reminded that the game the agents play only models the agents expected outcomes, and it does not have to correspond to the realization of the $\mathrm{CA}$ evacuation model.

Next, a step-by-step description is given of the spatial game coupled to the CA evacuation model. In the beginning of the simulation, the agents are located randomly in the room.

Step 1. At the beginning of each time step, the game parameters $T_{i j}=\left(T_{i}+T_{j}\right) / 2, i \neq j, \forall i, j \in I$ are calculated according to Eq. (1).

Step 2. The agents' strategies are updated with the shuffle update scheme until an equilibrium is reached. The agents observe the strategies of the other agents in their Moore neighborhood, and choose a best-response strategy according to Eq. (4).

Step 3. The agents' behavior in the CA model is set to correspond their strategy choice. This is done by altering $k_{S F}$ for the agents as follows:

(a) For an agent playing Impatient $k_{S F}=10.0$.

(b) For an agent playing Patient $k_{S F}=1.0$.

Step 4. Friction parameter $\mu$ is calculated according to Eq. (8).

Step 5. The agents' positions are updated in parallel according to Eq. (7). In a case of a conflict, one of the agents is allowed to move with probability $1-\mu$.

Step 6. Go to Step 1. The procedure is repeated until all agents have evacuated the room.

\section{Simulation results}

We are interested in how the agent's type affects the efficiency of the evacuation on both individual and crowd level. Thus, in Sec. 5.1, we simulate an evacuation with both high and low $T_{A S E T}$ agents, to see how the different agent types perform against each other. And, in Sec. 5.2, we simulate an evacuating crowd consisting of a single type agent, to see how the different types of agents perform on a crowd level, and show that the faster-is-slower effect is a result of agents' subjective interpretation of the threat. In the last two experiments, we study the mechanism behind the faster-is-slower effect. In Sec. 5.3, we look at the time evolution of a single simulation, to find that the agents evacuate in a stepwise manner. Finally, in Sec. 5.4 we study the distribution of the step lengths, or time lapses between consecutively evacuated agents. 
In the simulations, we are using a crowd of 200 agents. Thus, smaller values for $T_{A S E T}^{H}$ and $T_{A S E T}^{L}$ are used in comparison to the equilibrium simulations in Sec. 3. We set $T_{A S E T}^{H}=120$ and $T_{A S E T}^{L}=30$. The capacity of the exit is $\beta=1.25(1 / s)$, in all simulations. For impatient agents the coupling parameter is set to $k_{S F}=10$ and for patient agents $k_{S F}=1$. In all simulations, a one cell, i.e., $0.4 \mathrm{~m}$ wide exit is used. The friction parameter is of the form $\mu=0.6 \rho_{a} \rho_{\text {Imp }}+0.2 \rho_{a}+0.2 \rho_{\text {Imp }}$.

Sensitivity analysis on the effect of $\mu$ on macroscopic quantities of an evacuation simulation, e.g., flow at exit, have been already studied thoroughly in $[12,16]$. Thus, the authors are left with the following conclusion: it appears that the faster-is-slower effect is not sensitive to changes in $b_{1}$, as long as it is not decreased too much from 0.6. The faster-is-slower effect is more sensitive to the values of $b_{2}$ and $b_{3}$. They should not be set much higher than 0.2 for the model to work.

\subsection{Performance of the individuals}

Next, we study how high $T_{A S E T}$ agents perform against low $T_{A S E T}$ agents. In Fig. 5, a crowd of 200 agents, with 100 high $T_{A S E T}$ agents, and 100 low $T_{A S E T}$ agents, have been set in a room to evacuate through a narrow exit. The simulation was run 100 times, and the number of evacuated agents as a function of time was monitored. The gray curve represents the average number of evacuated high $T_{A S E T}$ agents, and the black curve the average number of evacuated low $T_{A S E T}$ agents.

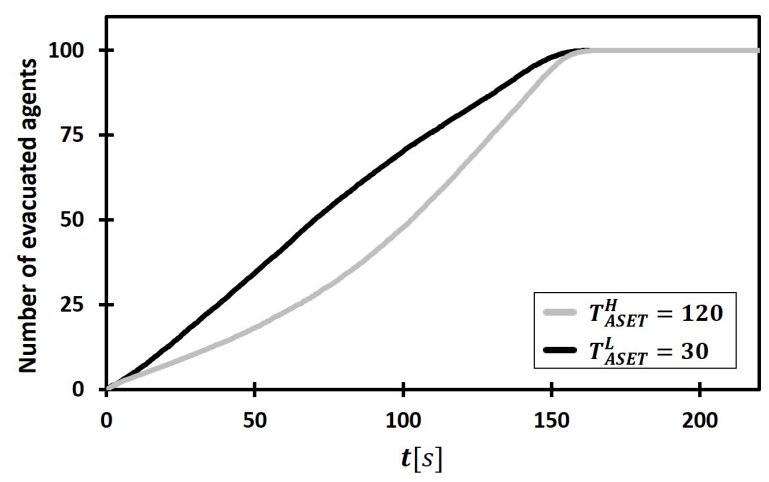

Figure 5: Evacuation of a crowd consisting of 100 high $T_{A S E T}$ agents and 100 low $T_{A S E T}$ agents. Averaged number of evacuated agents as a function of time for both agent types.

In Fig. 5, it can be seen that the average number of evacuated low $T_{A S E T}$ agents is almost always higher, than the average number of evacuated high $T_{A S E T}$ agents. However, both types of agents have evacuated at about the same point in time. Thus, our simulations show that the majority of low $T_{A S E T}$ agents are able to rush to the exit before high $T_{A S E T}$ agents.

Since, at the beginning of each simulation, the agents' positions were randomized, this shows that on average low $T_{A S E T}$ agents are able to overtake high $T_{A S E T}$ agents in our simulations. This can be explained by the analyses from Sec. 3, which show that a low $T_{A S E T}$ is more prone to be impatient. Furthermore, in [16] it has been shown that individual impatient agents are able to overtake patient agents in the CA evacuation model.

\subsection{Performance of the crowd}

Next, we study how the agent types perform on a crowd level. We set all agents in a crowd to be of the same type, and see which crowd evacuates faster. Otherwise we take the same simulation setup as in Fig. 5. Two scenarios are simulated; in the first the crowd consists of high $T_{A S E T}$ agents, and in the second, it consists of low $T_{A S E T}$ agents. The simulations are run 100 times, and the results are seen in Fig. 6.

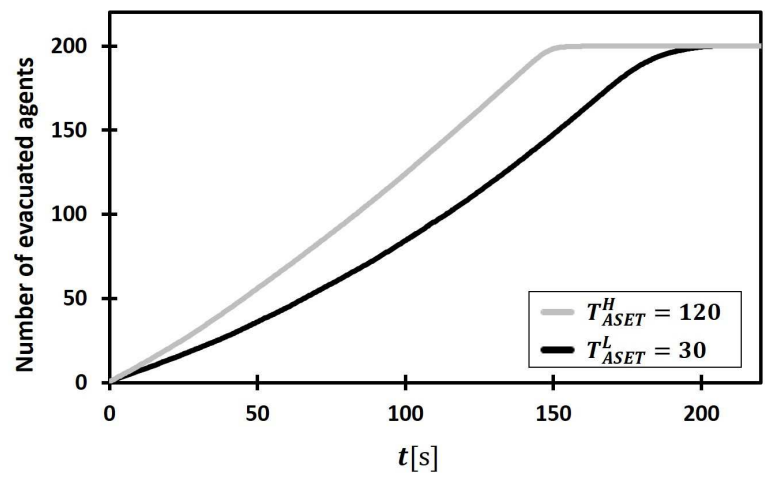

Figure 6: Averaged number of evacuated agents as a function of time for two different values of $T_{A S E T}$.

The gray curve represents the average number of evacuated high $T_{A S E T}$ agents, and the black curve the average number of evacuated low $T_{A S E T}$ agents. Whereas an individual low $T_{A S E T}$ agent was able on average to evacuate faster than an individual high $T_{A S E T}$ agent in a twotype crowd, here the crowd with low $T_{A S E T}$ agents evacuate slower than a crowd with high $T_{A S E T}$ agents. As the crowd consists of only low $T_{A S E T}$ agents, there is nobody to overtake, and the agents just hinder each others' attempt to escape.

\subsection{Time evolution of an evacuation}

Because the values in Fig. 6 are averaged, the curves do not tell us about the development of a single simulation. In 
Fig. 7 we take a look at the first 40 seconds of a single simulation of the evacuation of both a crowd with only high $T_{A S E T}$ agents, and a crowd with only low $T_{A S E T}$ agents.

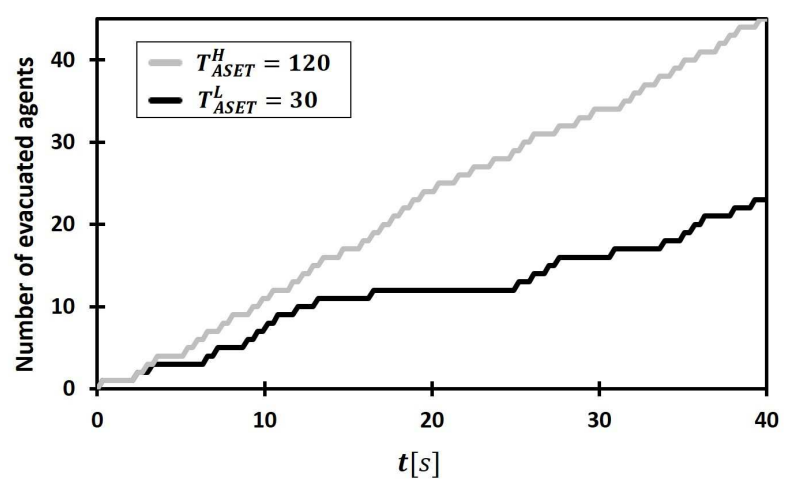

Figure 7: The development of a single simulation. Number of evacuated agents as a function of time for both agent types.

It is interesting to note that the curves, for both agent types, increase in an irregular stepwise manner. Actually, for a real crowd it is quite typical that people evacuate in such irregular successions [5]. In real crowds, it is a result of human arches forming and breaking down [6]. It is quite fascinating that the simple CA model can simulate this phenomenon. This feature of the $\mathrm{CA}$ was already noted in [12].

Though, in the CA, the phenomenon is not a result of human arches forming and breaking down, because no actual physical forces in the crowd are modeled. In the $\mathrm{CA}$, in front of the exit, there are constantly conflict situations. Whether any of the agents is allowed to move to the desired cell, is a consequence of the friction parameter $\mu$. The stochastic nature of these conflict situations introduces irregularity to the time lapses between consecutively evacuated agents. In [12] an analytical dependence between $\mu$ and the number of evacuated agents with respect to time has been derived.

The average amount of evacuated agents is indirectly proportional to the average time lapse between two consecutive agents. The step lengths of the curves show how long the time lapse is between two consecutive evacuated agents. Judging from Fig. 7, for the crowd with low $T_{A S E T}$ agents, the step lengths are longer, and there is more variability in their lengths.

\subsection{Distribution of time lapses}

Next, we calculate the average time lapses between two consecutive agents for both a crowd of only high $T_{A S E T}$ agents, and a crowd of low $T_{A S E T}$ agents. Since the friction parameter $\mu$ gives the probability that none of the agents in a conflict situation is allowed to move, the length of the time lapses is dependent on the value of $\mu$. Recall Eq. (8); $\mu$ depends on the size of the crowd. Thus, the distribution of the time lapses should depend on the size of the crowd. As the crowd is evacuating, the size of the crowd changes with time. Thus, we use data of only the 10 first time lapses from 100 simulations, which results in 1000 data points. For high $T_{A S E T}$ agents, the mean is 5.40 $\mathrm{s}$ and for low $T_{A S E T}$ agents it is $3.51 \mathrm{~s}$.

In Fig. 8 the complementary cumulative frequency distribution (complementary CDF) of the time lapses is plotted for a crowd with high $T_{A S E T}$ agents and for a crowd with low $T_{A S E T}$ agents. The complementary CDF equals $1-C D F$, and tells the probability of the time lapse, say $\triangle x$, being larger than some specific time $t$. Note that both of the axes are in logarithmic scale.

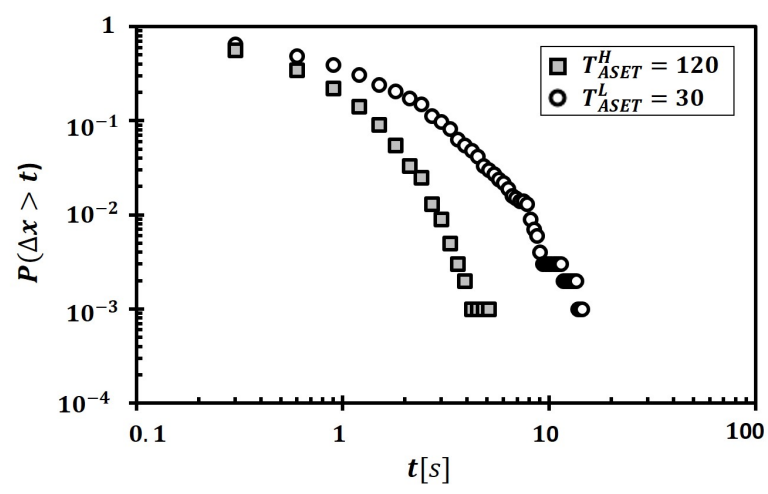

Figure 8: Complementary cumulative frequency distribution of the time lapses $\triangle x$ between two consecutive evacuated agents for both a crowd with high $T_{A S E T}$ agents and a crowd with low $T_{\text {ASET }}$ agents.

Note that the probability for low $T_{A S E T}$ agents is always larger than the probability for high $T_{A S E T}$ agents. Thus, our simulations show that longer time lapses are more probable for a crowd with low $T_{A S E T}$ agents.

\section{Conclusions}

The contribution of our article compared to previous articles $[7,16]$ is that here we have extended the spatial multiagent game, originally presented in [7] with only one agent type, to allow two types of agents, high and low $T_{A S E T}$ agents, and given a new explanation to the fasteris-slower effect with this model.

In Sec. 3, the equilibria of the game were studied. It was very interesting to notice that an agent, in a crowd with only high $T_{A S E T}$ agents, would be impatient in a certain location, but in a crowd with two types of agents, in exactly the same location, the agent would be patient. This can be explained by the low $T_{A S E T}$ agents' best-response 
strategy allowing a larger proportion of impatient agents in their neighborhood, compared to high $T_{A S E T}$ agents' best-response strategy. Thus, in a twotype crowd, in an equilibrium, it is the low $T_{A S E T}$ agents that are going to fill most of the spaces for impatient agents.

In Sec. 4, the spatial game was coupled to a modified version of the CA evacuation model from [16]. The dynamic floor field, which was included in the original CA evacuation model, was omitted, as its main purpose was to model the herding behavior of agents. The friction parameter $\mu$, which describes the probability that none of the agents in a conflict situation is able to move, was set to depend on the density of the crowd and proportion of impatient agents in it, thus more realistically modeling build-up of local pressure in the crowd.

In Sec. 5, evacuation of a crowd from a room was simulated. It was shown that low $T_{A S E T}$ agents were on average able to overtake high $T_{A S E T}$ agents. However, if all the agents in the crowd were low $T_{A S E T}$, they evacuated slower than a crowd were all the agents were high $T_{A S E T}$. Also, the underlying mechanism of the faster-is-slower effect was studied, by examining distribution of time lapses between consecutive agents. It was shown that the average time lapse between consecutively evacuating agents is higher for a crowd with low $T_{A S E T}$ agents. Moreover, it seems that longer time lapses are more probable for a crowd with low $T_{A S E T}$ agents. Our results coincide nicely with the experimental results in [5], even though we were not able to fit a power law to the tail of the complementary CDF.

In this article, we restricted ourselves to the simple scenario of a crowd evacuating from a room through a single exit. When modeling more complex geometries, we should take into account agents' exit selection and herding effects, i.e., that people go where the majority of the crowd is heading. Also, the static floor field and friction parameter $\mu$ should be set suitable for the new geometry.

\section{References}

[1] A. Kirchner, A. Schadschneider, Simulation of evacuation processes using a bionics-inspired cellular automaton model for pedestrian dynamics, Physica A: Statistical Mechanics and its Applications, 312 (2002) 260-276.

[2] R. Brown, Social Psychology. Free Press, New York, 1965.

[3] J. S. Coleman, Foundations of Social Theory. Harvard University Press, 1994.

[4] L. Y. Cooper, Compartment fire-generated environment and smoke filling, in SFPE Handbook of Fire Protection Engineering, National Fire Pritection Association (2002).
[5] A. Garcimartin, I. Zuriguel, J. M. Pastor, C. MartínGómez, D. R. Parisi, Experimental evidence of the "Faster Is Slower" effect, in Proc. Transportation Research Procedia (2014) 760-767.

[6] D. Helbing, Simulating dynamical features of escape panic, Nature, 407 (2000) 487-490.

[7] S. Heliövaara, H. Ehtamo, D. Helbing, T. Korhonen, Patient and impatient pedestrians in a spatial game for egress congestion, Physical Review E, 87 (2013) 012802.

[8] S. Heliövaara, J. M. Kuusinen, T. Rinne, T. Korhonen, H. Ehtamo, Pedestrian behavior and exit selection in evacuation of a corridor-An experimental study, Safety Science, 50 (2012) 221-227.

[9] S. P. Hoogendoorn, P. HL. Bovy, Simulations of pedestrian flows by optimal control and differential games, Optimal Control Applications and Methods, 24 (2003) 153-172.

[10] N. R. Johnson, Panic and the breakdown of social order: Popular myth, social theory, empirical evidence, Sociological Focus, 20 (1987) 171-183.

[11] H. H. Kelley, J. C. Condry, A. E. Dahlke, A. H. Hill, Collective behavior in simulated panic situation, Journal of Experimental Social Psychology, 1 (1965) 20-54.

[12] A. Kirchner, K. Nishinari, A. Schadschneider, Friction effects and clogging in a cellular automaton model for pedestrian dynamics, Physical Review E, 67 (2003) 056122.

[13] A. Mintz, Non-adaptive group behavior, The Journal of Abnormal Social Psychology, 46 (1951) 150-159.

[14] H. Muir, A. Cobbett, Cabin crew behavior in emergency evacuations, Civil Aviation Authority/Federal Aviation Administration Paper DOT, FAA/CT-95/16 (1995).

[15] X. Pan, C. S. Han, K. Dauber, K. H. Law, A multi-agent based framework for the simulation of human and social behavior during emergency evacuations, Ai \& Society, 22 (2007) 113-132.

[16] A. von Schantz, H. Ehtamo, Spatial game in cellular automaton evacuation model, Physical Review E, 92 (2015) 052805 .

[17] A. von Schantz, H. Ehtamo, Cellular automaton evacuation model coupled with a spatial game, in Proc. Active Media Technology, (2014) 371-382.

[18] J. M. Smith, Evolution and the Theory of Games, Cambridge University Press, 1982.

[19] G. Szabó, G. Fath, Evolutionary games on graphs, Physics Reports, 446 (2007) 97-216. 